\title{
Proliferating Trichilemmal Tumor Presenting as a Scrotal Mass in a Middle-Aged Male: An Uncommon Location
}

\author{
Aldulescu $\mathbf{M}^{1 *}$, Pins $\mathbf{M}^{2}$, and Speiser $\mathrm{J}^{1}$ \\ ${ }^{1}$ Department of Pathology, Loyola University Medical Center, USA \\ ${ }^{2}$ Department of Pathology, Lutheran General Hospital, USA
}

*Corresponding author: Dr. Monica Aldulescu DO, Department of Pathology, Loyola
Case Report

Volume 2 Issue 2

Received Date: June 03, 2017

Published Date: June 15, 2017

DOI: $10.23880 /$ cdoaj 16000124

University Medical Center, 2160 S. 1st Avenue, Maywood, IL 60153, USA, Tel: 708-327-2626; E-mail: Monica.Aldulescu@lumc.edu

\section{Abstract}

Scrotal masses encompass a heterogenous group of conditions affecting the skin and soft tissue of the scrotum or underlying testis, ranging from benign to malignant and from indolent to conditions requiring urgent medical evaluation. Accurate and timely diagnosis is imperative with scrotal masses, as the medical ramifications of different diagnoses vary widely, and the social and psychological implications can be significant. Here we present the first reported case of a middle-aged male with an enlarging scrotal/extra-testicular mass, which upon excision, was found to be consistent with a proliferating trichilemmal tumor, a benign neoplasm derived from outer root sheath of the hair follicle which has a strong capacity to simulate squamous cell carcinoma. Distinguishing between these two entities is of utmost importance due to their significantly different prognostic and treatment ramifications, and ultimately directs appropriate management of these patients.

Keywords: Scrotum; Testicle; Testis; Testes; Squamous cell carcinoma; Male; Proliferating; Trichilemmal tumor; Neoplasm

\section{Introduction}

Scrotal masses encompass a heterogeneous group of conditions affecting the skin and soft tissue of the scrotum or underlying testis, ranging from benign to malignant and from indolent to conditions requiring urgent medical evaluation [1]. Accurate and timely diagnosis is imperative with scrotal masses, as the medical ramifications of different diagnoses vary widely, and the social and psychological implications can be significant. Benign conditions affecting the scrotum and testes include, but are not limited to, epidermal cyst, inguinal hernia, epididymitis, orchitis, spermatocele, hydrocele, varicocele, hematocele, and testicular torsion [1]. On the opposite end of the spectrum are malignant tumors of the scrotum and testes. While a variety of seminomatous and non-seminomatous germ cell tumors are the most common malignancies in the testes, squamous cell carcinoma (SCC) remains the most common malignancy of the scrotum [2]. SCC of the scrotum has been a topic of interest for both clinical and historical reasons. It was documented by Pott in 1775 as one of the first occupational diseases occurring as a result of chronic soot exposure in the rugal folds of chimney sweeps [3-5]. While now rare due to improved working conditions, SCC still remains a risk in a variety of industrial occupations such as car and airplane manufacturing / repair, gas distribution, engineering, textile milling and metalworking [4,6-8]. Although occupational SCC is likely due to exposure to carcinogenic polycyclic aromatic 


\section{Clinical Dermatology Open Access Journal}

hydrocarbons, its incidence overall remains high as new risk factors, such as phototherapy for cutaneous conditions and human papilloma virus (HPV) have emerged [5]. The most common clinical and histologic differential diagnoses for SCC of the scrotum include epidermal inclusion cyst, extramammary Paget's disease, basal cell carcinoma, melanoma, and adnexal skin tumors [9]. This paper aims to add one more entity to this differential: proliferating trichilemmal tumor (PTT), as it can be clinically and histologically confused with the more common SCC of the scrotum, and has significantly different prognostic and treatment ramifications.

\section{Case Report}

We report the case of a 48 year-old male, who presented to his primary care physician with a progressively enlarging scrotal mass of unknown duration. He was referred to urology who tested tumor markers for LDH, AFP and beta-HCG, all of which were within normal limits. A scrotal ultrasound was performed which showed a mass of "mixed echogenicity," however it was uncertain whether the tumor showed testicular or spermatic cord involvement. It was not until intraoperative exploration that it was determined that both the spermatic cord and testis were in fact uninvolved. Upon excision, the specimen consisted of an ellipse of skin with a unilocular flesh-colored mass with cystic areas showing a smooth, focally trabeculated lining, as well as a $1.3 \mathrm{~cm}$ solid, fleshy, mural nodule (Figure 1). Microscopic sections showed a well-circumscribed dermal neoplasm with no connection to the overlying epidermis and a pushing boarder (Figure 2). It was comprised of a large cyst lined by stratified squamous epithelium devoid of a granular layer with peripheral palisading and trichilemmal-type keratinization (Figure 3) and a solid component consisting of interanastomosing lobules of squamous epithelium and cystic structures, devoid of a granular layer with foci of trichilemmal-type keratinization (Figure 4) [1,2]. Higher power examination revealed no significant cytologic atypia, necrosis, atypical mitoses or infiltration into the surrounding tissue (Figure 5). The differential diagnosis included squamous cell carcinoma, however immunohistochemical stain for Ki-67 showed a mitotic count of less than $5 \%$. Furthermore, immunostaining for CD34 and calretinin were both positive, confirming outer root sheath origin (Figures 6 and 7). Given the findings above, including lack of infiltration into the surrounding tissue, lack of significant cytologic atypia or mitoses, lack of necrosis, and presence of outer root sheath differentiation evident on CD34 and calretinin immune staining, a diagnosis of proliferating trichilemmal tumor (PTT) was rendered.

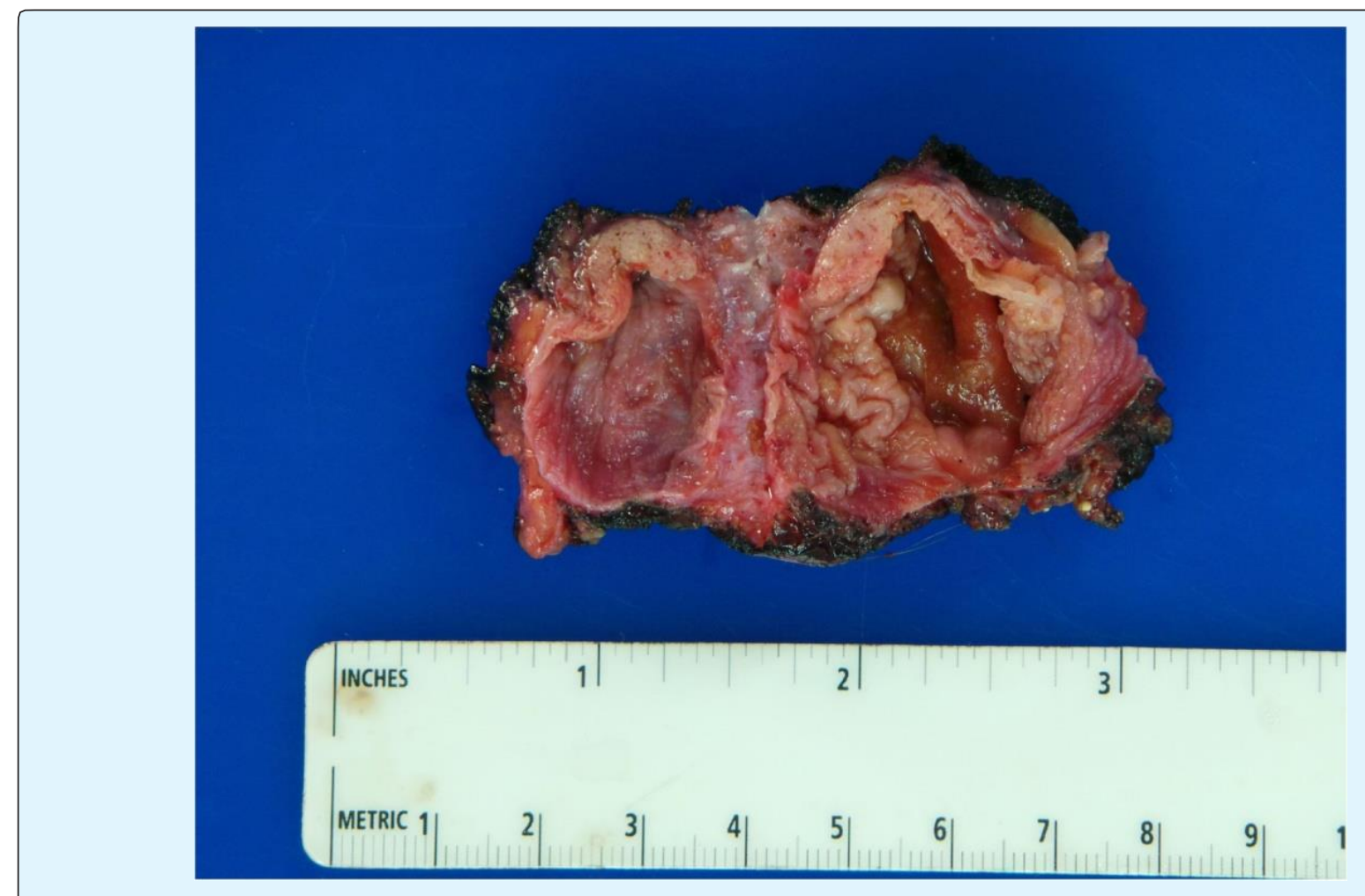

Figure 1: Gross Specimen. Bisected scrotal cyst showing a smooth, focally trabeculated, hemorrhagic cyst lining. 


\section{Clinical Dermatology Open Access Journal}

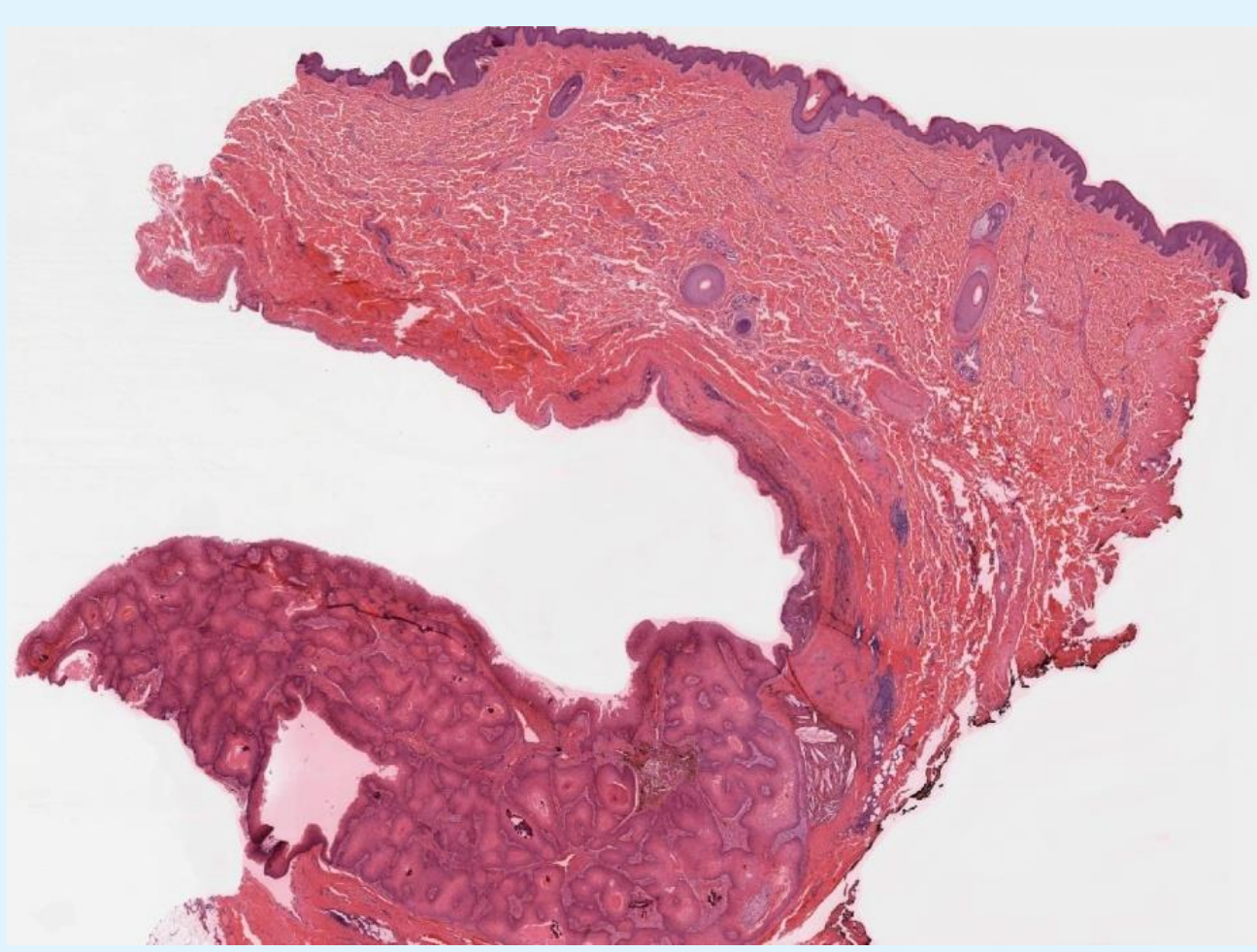

Figure 2: H\&E, 20x. Low power view shows a well-circumscribed dermal neoplasm with no connection to the overlying epidermis and a pushing boarder.

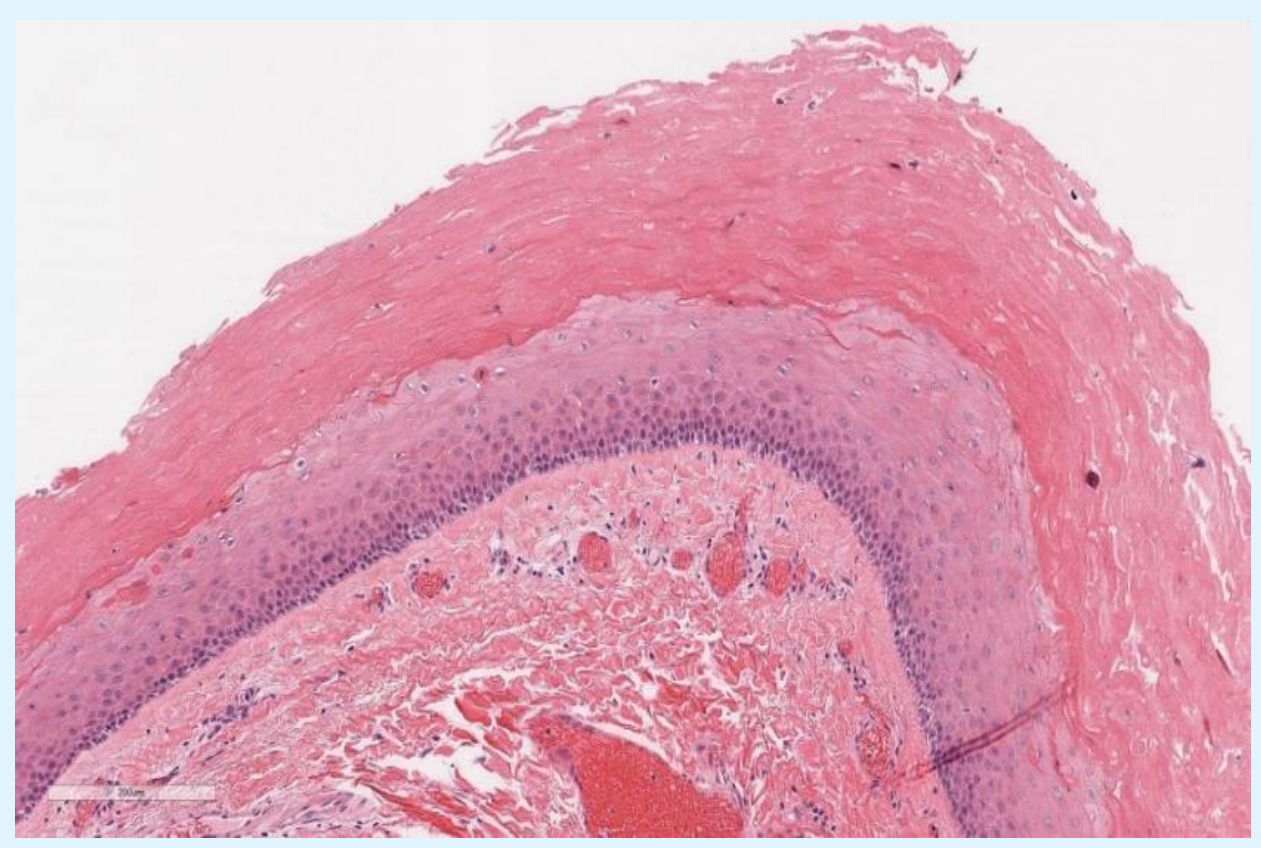

Figure 3: H\&E, 200x. High power view shows a portion of the cyst wall, lined by stratified squamous epithelium devoid of a granular layer with peripheral palisading and trichilemmal-type keratinization. 


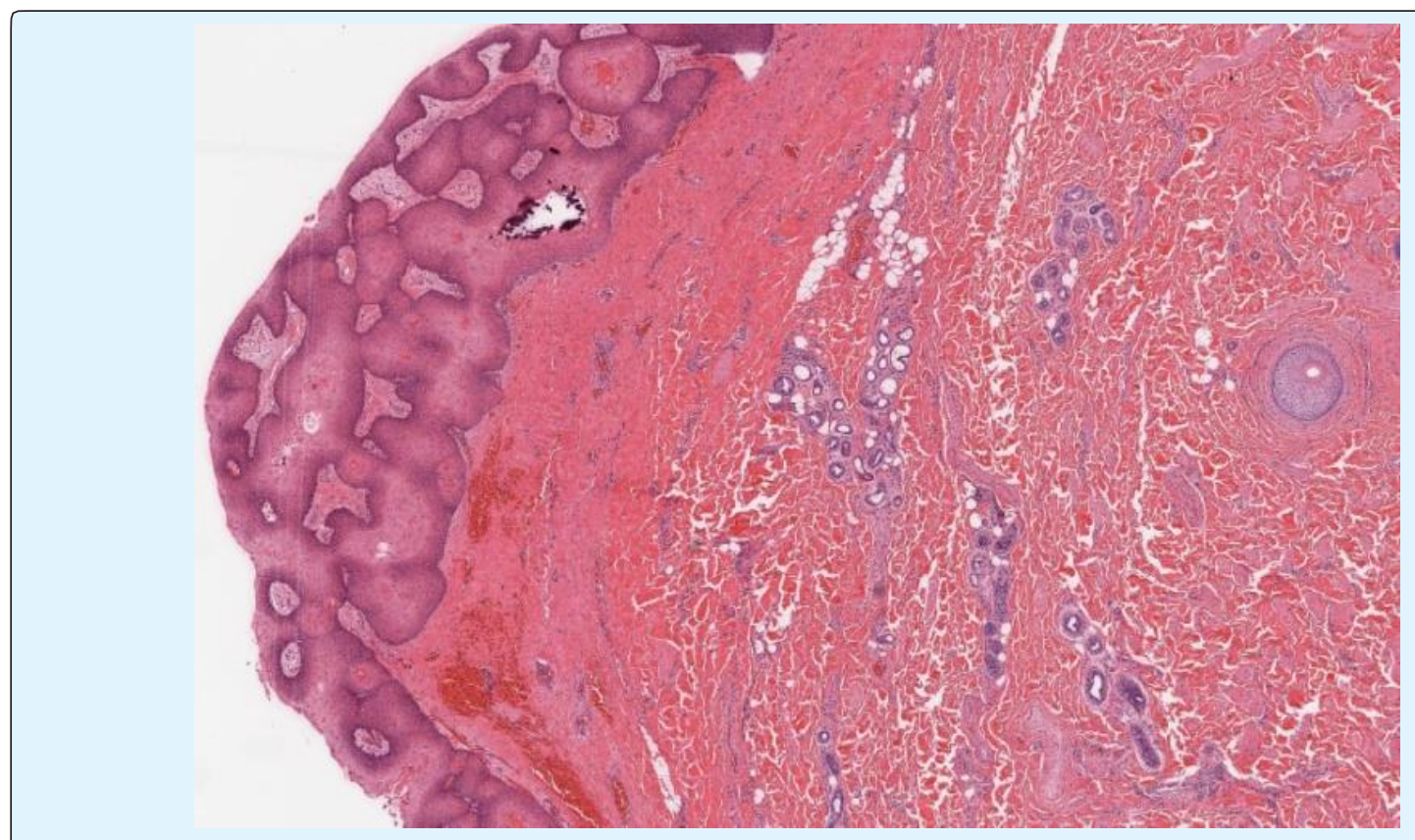

Figure 4: H\&E, 200x. High power view shows the solid component, which consists of interanastomosing lobules of squamous epithelium devoid of a granular layer with foci of trichilemmal-type keratinization.

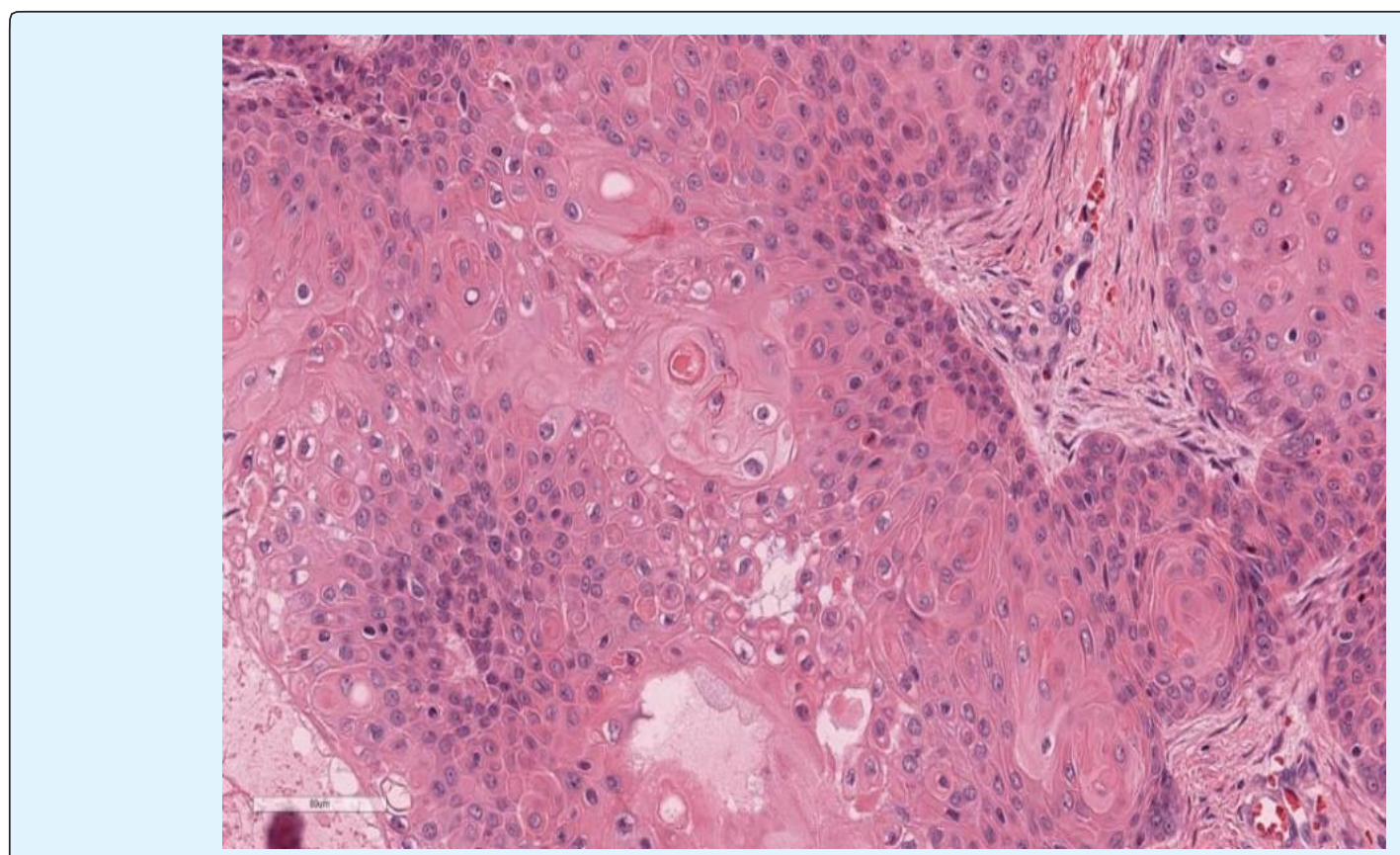

Figure 5: H\&E, 400x. High power view shows no significant cytologic atypia, necrosis, atypical mitoses, or infiltration into the surrounding tissue. 


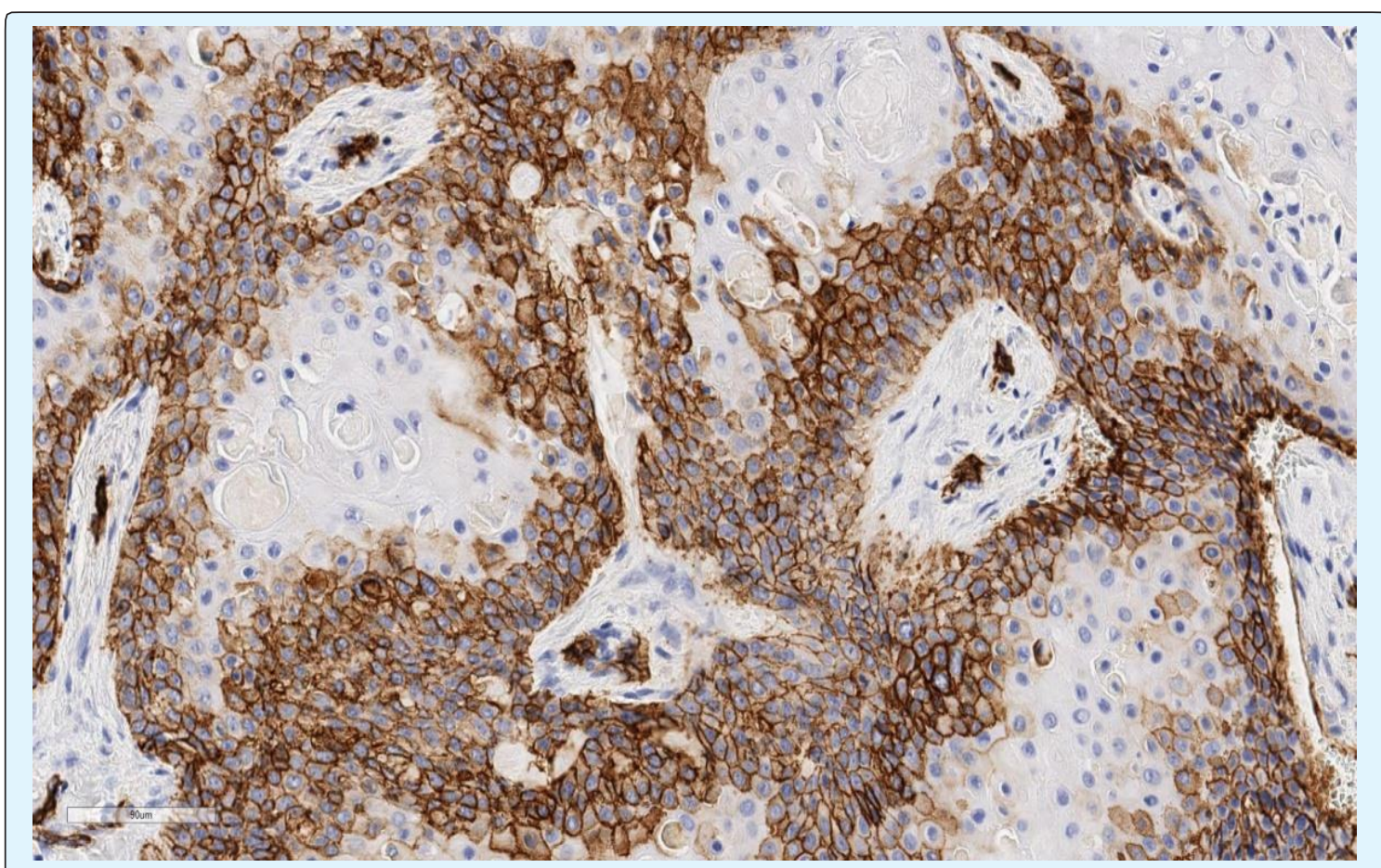

Figure 6: CD34, 400x. High power view shows positive membrane staining with normal vascular endothelial cells acting as internal control.

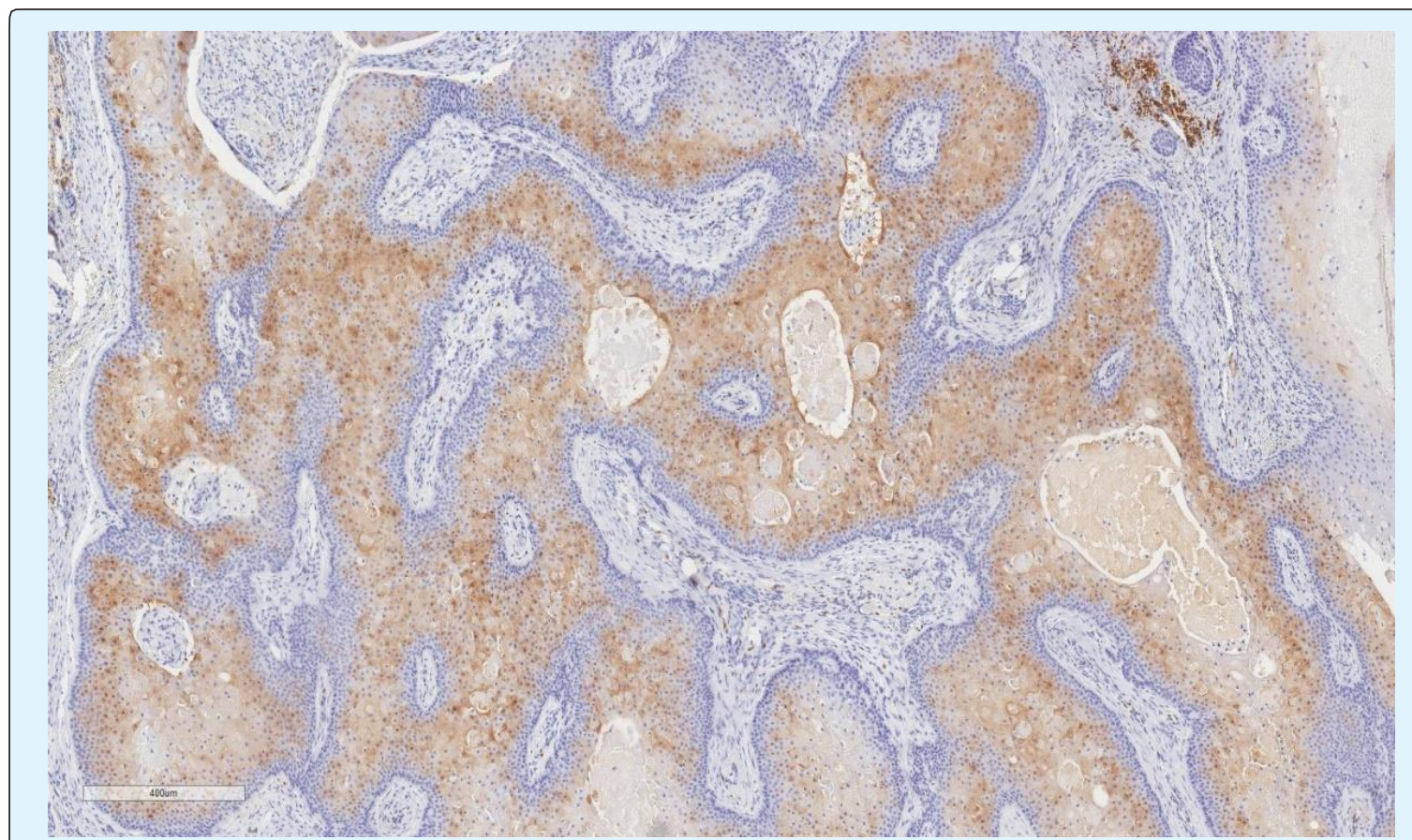

Figure 7: Calretinin, 200x. High power view of calretinin immunostain shows positivity in the tumor epithelium close to the lumen of the cystic structures in the PTT. 


\section{Clinical Dermatology Open Access Journal}

\section{Discussion}

Proliferating trichilemmal tumors (PTT) are uncommon and usually benign neoplasms arising from the isthmus region of the outer sheath of the roots of hair follicles. Wilson-Jones first described PTT in 1966 as an entity that can clinically and histologically simulate squamous cell carcinoma.20 Initially classified as a pseudoepitheliomatous hyperplasia, a number of other terms such as proliferating epidermoid cyst, pilar tumor of the scalp, proliferating trichilemmal cyst, proliferating epidermoid cyst, giant hair matrix tumor, hydatidiform keratinous cyst, and invasive hair matrix tumor have been used [10]. PTT typically presents as a solitary, painless nodule, ranging in size from 1 to $10 \mathrm{~cm}$. Although the majority (90\%) present on the scalp, other hair-bearing anatomic sites, including the trunk, and rarely the extremities, nose, eyelid and vulva, can also be involved [9]. There is a marked female predominance. They are characterized histologically by features of a typical pilar cyst, but additionally show extensive epithelial proliferation with a pushing boarder, mild cytologic atypia and mitotic activity [3]. Rarely, malignant transformation can occur, which demonstrates severe nuclear atypia, atypical mitoses, necrosis and an infiltrative growth pattern [11]. Surgical excision with a minimal negative margin is the treatment for PTT [11]. To our knowledge, this is the first reported case of a proliferating pilar cyst presenting in the scrotum.

In contrast, squamous cell carcinoma (SCC), although rare, remains the most common scrotal malignancy with a propensity for recurrence and metastasis [12]. It most commonly presents as a solitary erythematous nodule or plaque with or without keratinization or ulceration, most commonly on the left scrotum [1,2]. Histologically, squamous cell carcinoma consists of cords, strands and islands of squamous cells originating from the overlying epidermis and invades into the dermis with an infiltrative boarder [13]. There is significant cellular atypia, numerous atypical and typical mitoses, and variable necrosis. The cells have abundant eosinophilic cytoplasm, large vesicular and pleomorphic nuclei and prominent nucleoli [14]. Keratin pearls can be present throughout the tumor depending on its level of differentiation. Although surgical excision is also the primary treatment for SCC, current guidelines require a wider margin (range $4 \mathrm{~mm}-3 \mathrm{~cm}$ ) than PTT $[9,15]$. Additionally, due to the fact that the raphe of the scrotum does not provide a physical barrier to scrotal lymphatic drainage and that the scrotum has bilateral inguinal drainage, secondary treatments such as (1) inguinal sentinel lymph node dissection (ILND) / subsequent complete ILND in cases with clinical or histologic evidence for regional spread of disease and (2) palliative chemotherapy for locally advanced and metastatic disease are advocated for SCC $[15,2,16]$. It is important for clinicians to be aware of PTT as an entity when discussing the differential diagnosis of scrotal masses, as it can be clinically and histologically confused with the more common SCC of the scrotum and has significantly different prognostic and treatment ramifications.

\section{Conclusion}

PTTs are uncommon, slow-growing tumors that can pose a diagnostic dilemma due to their ability to mimic squamous cell carcinoma, especially when presenting in an unusual location. To our knowledge, this is the first case of a PTT described in the scrotum.

\section{References}

1. McDonald MW (1982) Carcinoma of scrotum. Urology 19(3): 269-74.

2. Southam AH, Wilson SR (1922) Cancer of the scrotum: The Etiology, Clinical Features, and Treatment of the Disease. Br Med J 2(3229): 971970.1.

3. Doll R (1975) Part III: 7th Walter Hubert lecture. Pott and the prospects for prevention. Br J Cancer 32(2): 263-272.

4. Doll R, Vessey MP, Beasley RW, Buckley AR, Fear EC, et al. (1972) Mortality of gas workers - final report of a prospective study. BrJ Industrial Med 29(4): 394406.

5. Kipling MD, Waldron HA (1975) Percivall Pott and cancer scroti. Br J Industrial Med 32(3): 244-246.

6. Fay HT (1978) Risk factors in scrotal epithelioma. J R Soc Med 71(10): 741-747.

7. Angulo JC, Lopez JI, Flores N (1993) Squamous cell carcinoma of the scrotum in an aluminium worker. Postgrad Med J 69(818): 960-961.

8. Cruickshank CN, Squire JR (1950) Skin cancer in the engineering industry from the use of mineral oil. Br J Ind Med 7(1): 1-11.

9. Azike JE (2009) A review of the history, epidemiology and treatment of squamous cell carcinoma of the scrotum. Rare Tumors 1(1): e17. 


\section{Clinical Dermatology Open Access Journal}

10. Bury Y, Bloxham C (2009) Profilerating trichilemmal tumour. Diagnostic Histopathology 15(5): 273-278.

11. Roy Sampurna (2016) Pathology of Proliferating Trichilemmal Tumour and Malignant Proliferating Trichilemmal Tumour.

12. Vyas R, Zargar H, Trolio RD, Lorenzo GD, Autorino R (2014) Squamous cell carcinoma of the scrotum: A look beyond the chimneystacks. World J Clin Cases 2(11): 654-660.

13. Barnhill Raymond L (2010) Dermatopathology $3^{\text {rd }}$ (Edn.), McGraw-Hill Medical Print, New York

14. Jackson R (1973) Early clinical descriptions of skin cancer. Can Med Assoc J 109(9): 906-908.

15. Motley R, Kersey P, Lawrence C, British Association of Dermatologists; British Association of Plastic Surgeons (2003) Multiprofessional guidelines for the management of the patient with primary cutaneous squamous cell carcinoma. Br J Plast Surg 56(2): 8591.
16. Lee S, Lee JH, Jeon SH, Kim MJ (2010) Multiple Epidermoid Cysts Arising from the Extratesticular Scrotal, Spermatic Cord and Perineal Area. Korean J Urol 51(7): 505-507.

17. Mohite P, Bhatnagar A (2006) A case of multiple sebaceous cysts over scrotum in a 35 years old male. Internet J Surg 9(1).

18. Gonzalez-Guerra E, Requena L, Kutzner H (2008) Immunohistochemical study of calretinin in normal hair follicles and tumors with follicular differentiation. Actas Dermosifiliogr 99(6): 456-463.

19. Poblet E, Jimenez F, Cabo C, Prieto-Matin A, SanchezPrieto R (2005) The calcium-binding protein calretinin is a marker of the companion cell layer of the human hair follicle. Br J Dermatol 152(6): 13161320.

20. Sharma R, Verma P, Yadav P, Sharma S (2012) Proliferating Trichilemmal Tumor of Scalp: Benign or Malignant, A Dilemma. J Cutan Aesthet Surg 5(3): 213-215. 